\title{
Polarization Dependence of Carrier Extraction from Dual-Polarization Phase-Coujugated Twin Waves with Sum-Frequency Generation
}

\author{
Yasuhiro OKAMURA, Ikuya NAGATOMI, and Atsushi TAKADA \\ Tokushima University, 2-1 Minamijosanjima, Tokushima, Tokushima 770-8506
}

(Received February 27, 2018)

\begin{abstract}
This study aimed to build a numerical model of carrier extraction from dual-polarization phaseconjugated twin waves (DP-PCTWs) to investigate polarization dependence on the carrier extraction in a pump phase-locked loop for phase-sensitive optical amplifier repeaters. First, the numerical model was developed, and extracted carrier power of 40 - Gbit/s DP-PCTWs with quadrature phase-shift keying format was calculated. Numerical results show the extracted carrier disappears when the PCTWs are circular polarization, and polarization dependence exists on the carrier extraction. Then, carrier extraction of a 10- Gbit/s DP-PCTW with binary phase-shift keying format was experimentally demonstrated. The extracted carrier power was found to be relatively low when the DP-PCTWs are circular polarization, and this trend agrees closely with the numerical results. These results show the reasonability of the numerical model and existence of polarization dependence on the carrier extraction from DP-PCTWs.
\end{abstract}

Key Words: Phase-sensitive optical amplifier, Optical phase-locked loop, Polarization dependence Carrier extraction

\section{Introduction}

It is clear that erbium-doped optical fiber amplifiers (EDFAs) have played an important role in modern optical fiber communication systems as amplifier repeaters to achieve high-capacity optical transmission. However, amplified spontaneous emission (ASE) resulting from the laser amplification process in EDFAs restricts transmission distance in spectrally efficient optical transmission systems with multi-level modulation formats such as $M$-ary quadrature amplitude modulation (QAM) because the required signal-to-noise ratio (SNR) increases as $M$ increases.

The frequency non-degenerate phase-sensitive optical amplifier (PSA), which is a kind of optical parametric amplifier, has recently received broad attention because of its attractive characteristics such as low-noise optical amplification and nonlinear phase noise suppression. ${ }^{1)}$ PSA repeaters are expected extend transmission distance in spectrally efficient optical transmission systems compared to conventional EDFA links. ${ }^{2)}$ In order to realize PSAs, phase-conjugated twin waves (PCTWs) $)^{3)}$ composed of a signal wave and its phase-conjugated wave, and a pump wave are incident on an optical nonlinear medium for nonlinear interaction between them, and pump phase-locking to the PCTWs is needed. There are two main methods to generate phase-synchronized pump waves for PSAs. One is an optical injection locking (OIL) method with pilot tone transmission. ${ }^{4)}$ The second is an optical phaselocked loop (OPLL) method. From the viewpoint of spectral efficiency, the pump phase-locked loop method ${ }^{5,6)}$ is the more promising candidate because a guard band is not required to mitigate the wavelength-channel crosstalk resulting from the pilot tone transmission.

We have proposed an optical phase-locked loop assisted by sum-frequency generation and second-harmonic generation
(SS-OPLL) for PSA repeaters. ${ }^{6}$ In SS-OPLL, the PCTW phase information is canceled by the sum-frequency generation (SFG) process, and a carrier is extracted. The second harmonic of a pump wave from an optical voltage-controlled oscillator (OVCO) is coupled to the extracted carrier, and the interference signal is detected by a balanced photodetector. Then, the phase error between the PCTWs and the pump is obtained and fed back to the OVCO. As a consequence, pump phase-locking to PCTWs is accomplished by the SS-OPLL. We have successfully demonstrated pump phase-locking to 80- $\mathrm{km}$ fiber-transmitted quadrature phase-shift-keying (QPSK) PCTWs in a single polarization channel system by the SS-OPLL and the phase-sensitive amplification of the PCTWs by using a phase-synchronized pump. ${ }^{7)}$ To further increase spectral efficiency, PSA repeaters must handle dual-polarization PCTWs (DP-PCTWs); PSA of DP-PCTWs has been demonstrated by using the OIL method in previous studies. ${ }^{89}$ ) In the case of the SS-OPLL, pump phase-locking to DPPCTWs with the SS-OPLL has not been demonstrated so far, and the SS-OPLL must be applied to DP-PCTWs to replace the OIL method with the SS-OPLL for elimination of the pilot tone transmission. In principle, when a carrier is extracted from DP-PCTWs by the SFG process, pump phase-locking to DP-PCTWs could be achieved. In fact, a periodically poled lithium niobate (PPLN) waveguide enables the SFG process, and such a device exhibits polarization dependence. However, a polarization of DP-PCTWs are varied through fiber transmission, and the polarization effect in the carrier extraction of DP-PCTWs has not been clarified yet.

In this study, we built a numerical model of carrier extraction from DP-PCTWs with an arbitrary polarization and investigated the influence of polarization on the carrier extraction from DP-PCTWs. First, a theoretically based model of SFG carrier extraction from DP-PCTWs with an arbitrary 
polarization was developed. Then, the polarization dependence of extracted carrier power due to the state of polarization (SOP) of a transmission fiber was calculated. Finally, carrier extraction from 10- Gbit/s DP-binary phase-shift keying (BPSK)-PCTWs with a circular polarization was experimentally demonstrated to confirm the validity of the built model and numerical results.

\section{Numerical Model of Carrier Extraction from DP- PCTWs by Second-Order Nonlinear Material}

A numerical model of SFG carrier extraction from DPPCTWs with an arbitrary polarization is shown in Fig. 1; the insets of Fig. 1 are signal power spectra in each section of the model. A continuous wave (CW) from a laser diode (LD) with optical frequency $v_{\mathrm{c}}$ is divided into two parts for $\mathrm{X}$ - and Y-polarization channel signal generation. In the upper arm, the $\mathrm{CW}$ is intensity-modulated by $\mathrm{X}$-ch. intermediate frequency phase-shift keyed (IF-PSK) signals $s_{\mathrm{X}}(t)=E_{\mathrm{IFCOS}}\left(2 \pi f_{\mathrm{IF}} t+\right.$ $\left.\theta_{\mathrm{dX}}\right)$ in which $E_{\mathrm{IF}}$ is the voltage amplitude, $f_{\mathrm{IF}}$ is the center frequency of the IF-PSK signals, and $\theta_{\mathrm{dX}}$ is the phase information of X-ch. Here, we assume that the optical intensity modulator with a half-wave voltage $V_{\pi}$ acts as product modulator. Then, PSK-PCTWs for X-ch. $e_{\mathrm{x}}(t)$ are generated and expressed by

$$
\begin{aligned}
& e_{\mathrm{X}}(t)=\frac{\pi E_{\mathrm{IF}} E_{\mathrm{c}}}{2 V_{\pi}} \\
& \times\left\{\begin{array}{l}
\exp j\left(\theta_{\mathrm{dX}}+\varepsilon_{\mathrm{c}}\right) \exp j\left\{2 \pi\left(v_{\mathrm{c}}+f_{\mathrm{IF}}\right) t\right\} \\
+\exp j\left(-\theta_{\mathrm{dX}}+\varepsilon_{\mathrm{c}}\right) \exp j\left\{2 \pi\left(v_{\mathrm{c}}-f_{\mathrm{IF}}\right) t\right\}
\end{array}\right\}
\end{aligned}
$$

Here, $E_{\mathrm{c}}$ indicates the electric field amplitude, and $\varepsilon_{\mathrm{c}}$ is the phase fluctuation of optical fields resulting from spontaneous emission of laser sources. The first term indicates signal waves, and the second term indicates phase conjugated waves. In the same fashion, Y-ch. PSK-PCTWs $e_{\mathrm{Y}}(t)$ with phase information $\theta_{\mathrm{dY}}$ are generated by using IF-PSK signals $s_{\mathrm{y}}(t)=$ $E_{\mathrm{IFCOS}}\left(2 \pi f_{\mathrm{IF}} t+\theta_{\mathrm{dY}}\right)$ for Y-ch., as shown in

$$
\begin{aligned}
& e_{\mathrm{Y}}(t)=\frac{\pi E_{\mathrm{IF}} E_{\mathrm{c}}}{2 V_{\pi}} \\
& \times\left\{\begin{array}{l}
\exp j\left(\theta_{\mathrm{dY}}+\varepsilon_{\mathrm{c}}\right) \exp j\left\{2 \pi\left(v_{\mathrm{c}}+f_{\mathrm{IF}}\right) t\right\} \\
+\exp j\left(-\theta_{\mathrm{dY}}+\varepsilon_{\mathrm{c}}\right) \exp j\left\{2 \pi\left(v_{\mathrm{c}}-f_{\mathrm{IF}}\right) t\right\}
\end{array}\right\} .
\end{aligned}
$$

PCTWs $e_{\mathrm{X}}(t)$ and $e_{\mathrm{Y}}(t)$ are polarization-multiplexed by a polarization beam splitter (PBS), and DP-PCTWs $\boldsymbol{e}_{\mathrm{PDM}}$ are generated.

$$
\boldsymbol{e}_{\mathrm{PDM}}=\left[e_{\mathrm{x}}(t) ; e_{\mathrm{Y}}(t)\right]
$$

The DP-PCTWs $\boldsymbol{e}_{\mathrm{PDM}}$ are input to a transmission fiber, and the polarization of the signals is varied. The SOP of the transmission fiber is represented by the Jones matrix $\mathbf{J}_{\text {Fiber }}(\phi, \theta)$ : ${ }^{10)}$

$$
\begin{aligned}
& \mathbf{J}_{\text {Fiber }}(\phi, \theta)=\left[\begin{array}{cc}
\exp \left(j \frac{\phi}{2}\right) \cos \frac{\theta}{2} & -\sin \frac{\theta}{2} \\
\sin \frac{\theta}{2} & \exp \left(j \frac{\phi}{2}\right) \cos \frac{\theta}{2}
\end{array}\right] \\
& =\left(\begin{array}{cc}
a & -b \\
b & a^{*}
\end{array}\right)
\end{aligned}
$$

$\phi$ and $\theta$ indicate the polar angle and azimuthal angle of the SOP on a Poincare sphere. After the transmission fiber, the DP-PCTWs with an arbitrary polarization can be represented as $\boldsymbol{e}_{\mathrm{PLLin}}=\mathbf{J}_{\mathrm{Fiber}}(\phi, \theta) \boldsymbol{e}_{\mathrm{PDM}}$ and are guided into a carrier extraction section in SS-OPLL. An X-polarization component of the DP-PCTWs passes through a polarizer with $\mathbf{J}_{\mathrm{Pol}}=[10$; 0 0] and input to a $\chi^{(2)}$ optical nonlinear medium for the SFG carrier extraction. Here, the input of the $\chi^{(2)}$ optical nonlinear medium $\boldsymbol{e}_{\mathrm{x} 2 \mathrm{in}}$ is described as $\boldsymbol{e}_{\mathrm{x} 2 \mathrm{in}}=\left[e_{\mathrm{x} 2 \mathrm{inX}}(t) ; e_{\mathrm{x} 2 \mathrm{in} Y}(t)\right]=\left[a e_{\mathrm{X}}(t)\right.$ $\left.-b e_{\mathrm{Y}}(t) ; 0\right]$, and only the X-polarization component of $\boldsymbol{e}_{\mathrm{x} 2 \mathrm{in}}$, i.e. $e_{\mathrm{x} 2 \mathrm{inX}}(t)$, is incident to the $\chi^{(2)}$ optical nonlinear medium. The output signal of the $\chi^{(2)}$ optical nonlinear medium with a conversion coefficient $k$ can be expressed by

$$
e_{\text {x2out }}(t)=k\left[\operatorname{Re}\left\{e_{\text {x2inX }}(t)\right\}^{2}\right]
$$

Finally, power spectrum density of the $\chi^{(2)}$ optical nonlinear medium output is calculated.

$$
E_{\text {x } 2 \text { PSD }}(f)=20 \log _{10}\left|F\left\{e_{\text {x } 2 \text { out }}(t)\right\}\right|
$$

$F\{\}$ denotes the Fourier transform operation. From this process, we can find the power spectrum density (PSD) of the carrier extracted from the DP-PCTWs.

\section{Polarization Dependence of Carrier Extraction from 40- Gbit/s DP-QPSK-PCTWs}

We calculated PSD of the extracted carrier from 40- Gbit/s DP-QPSK-PCTWs by the numerical model depicted in Fig. 1. In this calculation, the DP-QPSK-PCTWs with 100- GHz spacing are centrally located in $v_{\mathrm{c}}$, which is $193.41 \mathrm{THz}$, and employ a non-return-to-zero waveform. The linewidth of a

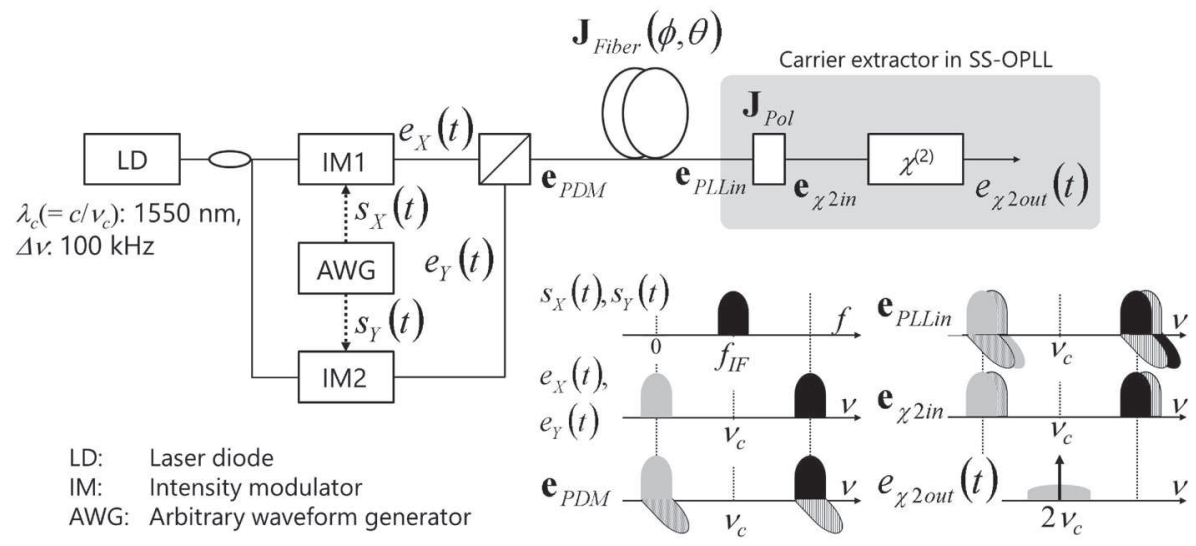

Fig. 1 Numerical model of carrier extraction from DP-PCTWs with a second-order nonlinear medium. 
signal light source is assumed to be $100 \mathrm{kHz}$. In the carrier extractor, the conversion coefficient of the $\chi^{(2)}$ optical nonlinear medium $k$ is set to 1 for simplicity. The azimuth angle $\theta$ and the polar angle $\phi$ in Eq. 4 range from 0 to $2 \pi$ for changing the SOP of the transmission fiber, and PSDs of the light field $e_{\mathrm{x} 2 \mathrm{out}}(t)$ at the output of the carrier extractor are found.

Calculated PSDs are shown as examples in Fig. 2. Figure 2 (a) shows the case of $(\phi, \theta)=(0, \pi / 2)$. The horizontal axis is frequency detuning from $2 v_{\mathrm{c}}$, and the vertical axis is normalized PSD. A line spectrum is located at $0 \mathrm{~Hz}$, and carrier extraction from the DP-QPSK-PCTWs is achieved in this SOP. Moreover, a modulation spectrum with $10-\mathrm{GHz}$ bandwidth is also centered at $0 \mathrm{~Hz}$. This is because a beat signal between a signal wave of the X-ch. PCTWs and a phase-conjugated wave of the Y-ch. PCTWs is generated through the SFG process. In addition, a beat signal between a phase-conjugated wave of the X-ch. PCTWs and a signal wave of the Y-ch. PCTWs is also generated. Such beat signals seem to deteriorate the extracted carrier; however, it is possible to use the carrier as a phase reference of the SS-OPLL because of its carrier-to-noise ratio of over $20 \mathrm{~dB}$. In the case of $(\phi, \theta)=(\pi$, $\pi / 2$ ), a carrier is not extracted as seen in Fig. 2 (b). In this case, the SS-OPLL does not work because there is no phase reference. These results show that there is polarization dependence on the carrier extraction from DP-PCTWs.

Then, polarization dependence of the extracted carrier power was investigated. Figure. 3 (a) shows normalized carrier power as a function of the polar angle $\phi$. When azimuth angle $\theta=\pi / 4$, $\pi / 2$, and $3 \pi / 4$, we found the minimum values were located at $\phi=$ $\pi$. The normalized carrier power as a function of the azimuth angle $\theta$ was also calculated as shown in Fig. 3 (b). When $\phi=\pi / 4$, $\pi / 2$, and $3 \pi / 4$, minimum values were found at $\theta=\pi / 2$ and $3 \pi / 2$.

A contour diagram of the extracted carrier power in a SOP determined by $\phi$ and $\theta$ is shown in Fig. 4. The color grade indicates the normalized carrier power. Two minimum values were found at $(\phi, \theta)=(\pi, \pi / 2),(\pi, 3 \pi / 2)$. In these cases, the Jones matrices of the transmission fiber $\mathbf{J}_{\text {Fiber }}(\phi, \theta)$ can be found as follows:

$$
\begin{aligned}
\mathbf{J}_{\text {Fiber }}(\pi, \pi / 2) & =\frac{1}{\sqrt{2}}\left[\begin{array}{cc}
j & -1 \\
1 & -j
\end{array}\right] \\
\mathbf{J}_{\text {Fiber }}(\pi, 3 \pi / 2) & =\frac{1}{\sqrt{2}}\left[\begin{array}{cc}
-j & -1 \\
1 & j
\end{array}\right]
\end{aligned}
$$

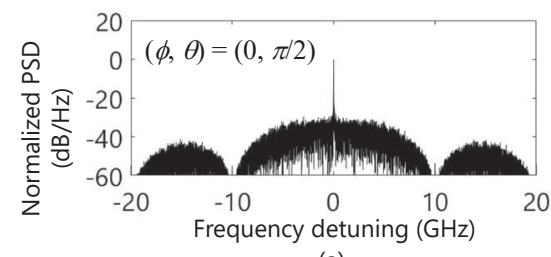

(a)

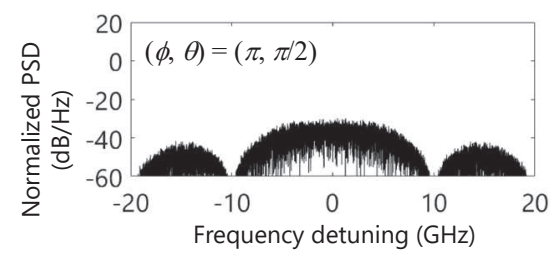

(b)

Fig. 2 Optical PSDs at the output of the second-order nonlinear medium. SOP of the transmission fiber $(\phi, \theta)$ is (a) $(0, \pi / 2),(\mathrm{b})(\pi, \pi / 2)$.

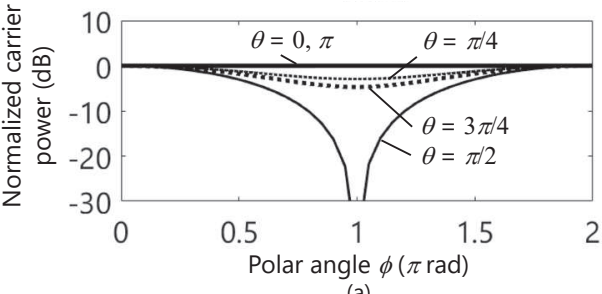

(a)

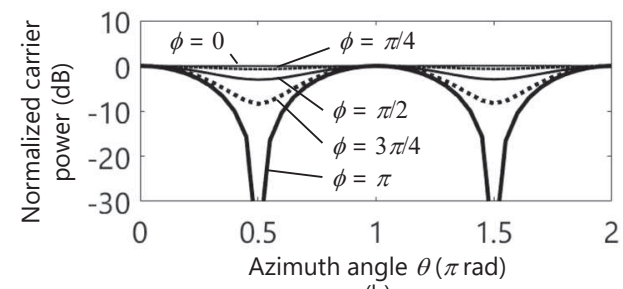

(b)

Fig. 3 Normalized carrier power as a function of (a) polar angle $\phi$ and (b) azimuth angle $\theta$.

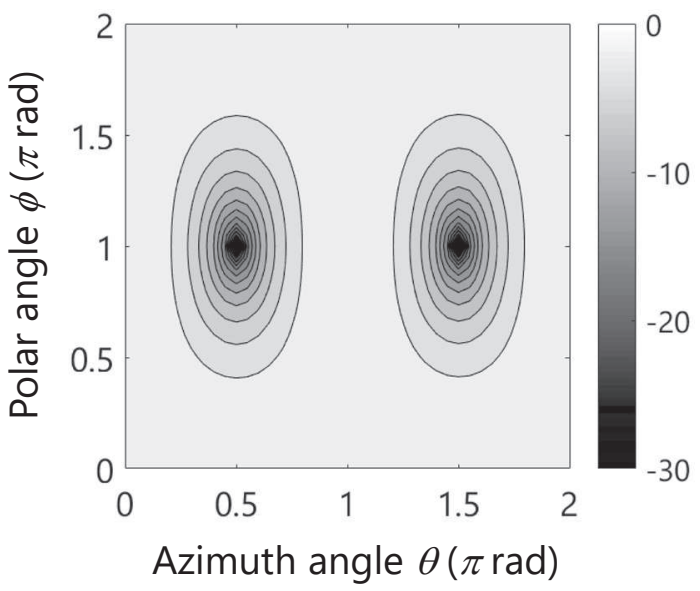

Fig. 4 Contour diagram of extracted carrier power for SOP $\mathbf{J}_{\text {Fiber }}(\phi, \theta)$. The color grade shows normalizedcarrier power.

Equations (7) and (8) indicate that when the carrier vanishes, the X-ch. PCTWs and the Y-ch. PCTWs have a phase difference of $\pi / 2$ and equal amplitude. It was assumed that after the SFG process, the extracted carriers from the X-ch. PCTWs and the Y-ch. PCTWs interfere with each other and are canceled out because the phase difference between the carriers becomes $\pi$.

\section{Experimental Verification with Carrier Extraction from 10- Gbit/s DP-BPSK-PCTWs}

Carrier extraction from 10- Gbit/s DP-BPSK-PCTWs was experimentally demonstrated to confirm the validity of the numerical model and the results. Figure. 5 shows the experimental setup. A CW emitting from an LD with 1552.5- nm wavelength and $2.0-\mathrm{kHz}$ linewidth was divided into two by a $10-\mathrm{dB}$ optical coupler. One was used for signal generation and the carrier extraction in the upper arm, and the other was used for heterodyne detection to measure the extracted carrier power in the lower arm. In the upper arm, BPSK-PCTWs were first generated. The incoming $\mathrm{CW}$ was intensity-modulated by a 5- Gbit/s BPSK signal with $18-\mathrm{GHz}$ IF that was generated by a signal generator and a pulse pattern generator. The output was input to an optical interleaver through an optical amplifier, and the residual carrier was suppressed. In this 


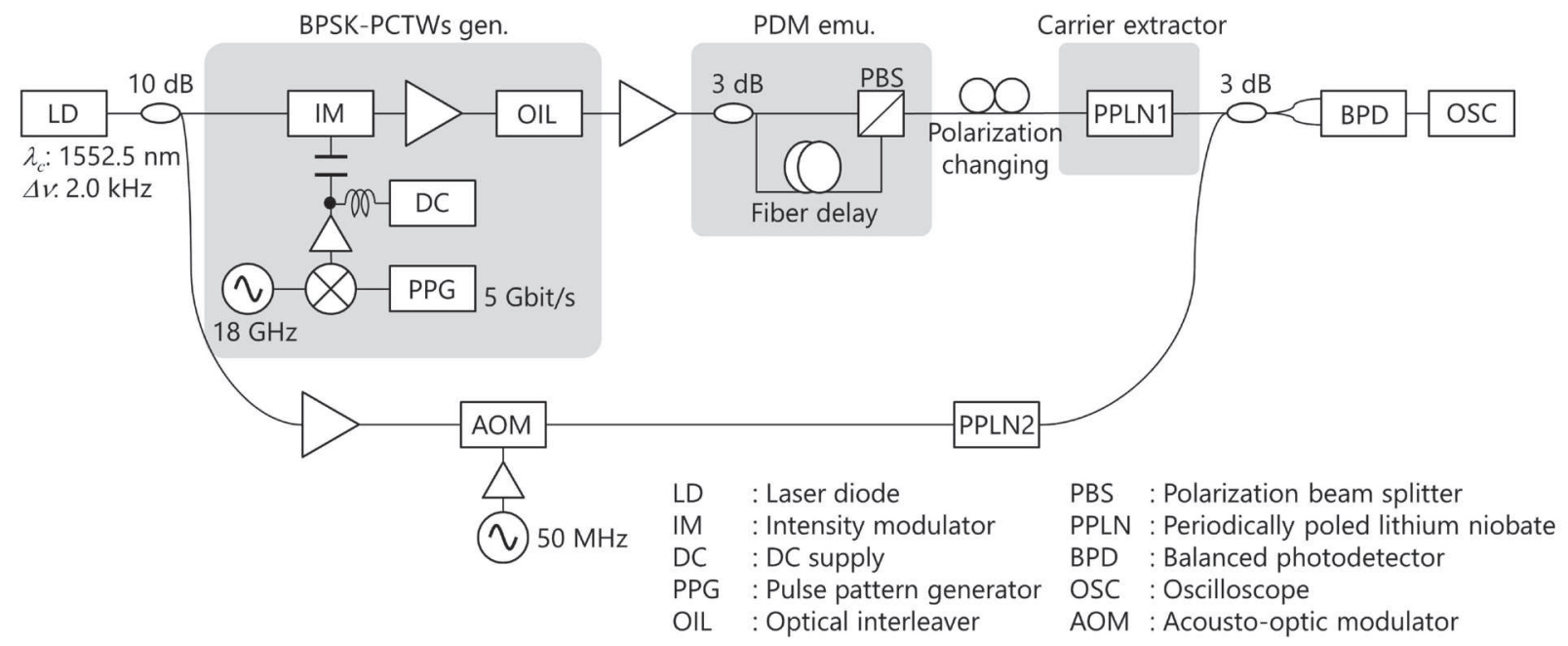

Fig. 5 Experimental setup of carrier extraction from 10- Gbit/s DP-BPSK-PCTWs.

manner, 5- Gbit/s BPSK-PCTWs were generated. Then, the BPSK-PCTWs were guided into a polarization division-multiplexed (PDM) emulation section. In the PDM emulator, the incoming BPSK-PCTWs were split by a 3-dB optical coupler. One part was delayed and polarization-multiplexed with another part by using a PBS. Thus, 10- Gbit/s DP-BPSKPCTWs were generated. The DP-BPSK-PCTWs were polarization-controlled by a polarization controller $\mathbf{J}_{\mathrm{PC}}$ composed of a half-wave plate (HWP) and a quarter-wave plate (QWP) and input to a PPLN1 for the carrier extraction. On the other hand, another light for the heterodyning was amplified and frequency-shifted by an acousto-optic modulator (AOM). The 50- MHz frequency-shifted CW was input to a PPLN2, and a second-harmonic (SH) wave with $100-\mathrm{MHz}$ frequency offset with respect to the extracted carrier was generated. The $\mathrm{SH}$ wave was interfered with the extracted carrier by a $3-\mathrm{dB}$ short-wavelength optical coupler, and a heterodyne beat signal with $100-\mathrm{MHz}$ frequency was observed by an oscilloscope (OSC) as a measurement of the extracted carrier power.

Figure 6 shows the observed waveform of the heterodyne beat signals. When the HWP was set to $45^{\circ}$, and the QWP was set to $0^{\circ}, \mathbf{J}_{\mathrm{PC}}=[0 \exp (j 3 \pi / 4) ; \exp (j \pi / 4) 0]$, and the only Y-ch. BPSK-PCTWs were input to the PPLN1. Therefore, a carrier was extracted from the Y-ch. PCTWs, and a 100- MHz beat signal with relatively large amplitude was observed as shown by the solid line. The extracted carrier power was $-14.5 \mathrm{dBm}$. In contrast, when the HWP was set to $0^{\circ}$, and the QWP was set to $45^{\circ}, \mathbf{J}_{\mathrm{PC}}=\left[\begin{array}{lll}j & 1 ; & -1\end{array}\right]$, DP-BPSK-PCTWs with circular polarization, in which the phase difference between the X-ch. and Y-ch. PCTWs was $\pi$, were input to the PPLN1. The result is shown by the dashed line in Fig. 6. The voltage amplitude is small compared to the $\mathbf{J}_{\mathrm{PC}}=[0 \exp$ $(j 3 \pi / 4) ; \exp (j \pi / 4) 0]$. The extracted carrier power was $-28.7 \mathrm{dBm}$ and lower than the previous case by $14.2 \mathrm{~dB}$. This trend agrees closely with the calculated results described in Section 3. Therefore, these results support the reasonability of the numerical model and the presence of polarization dependency on the carrier extraction from DP-PCTWs.

\section{Summary}

In this paper, a numerical model of the carrier extraction from DP-PCTWs for a pump phase-locked loop was built, and exhibited polarization dependence on the carrier extraction in the SS-OPLL. The numerical simulation results showed that

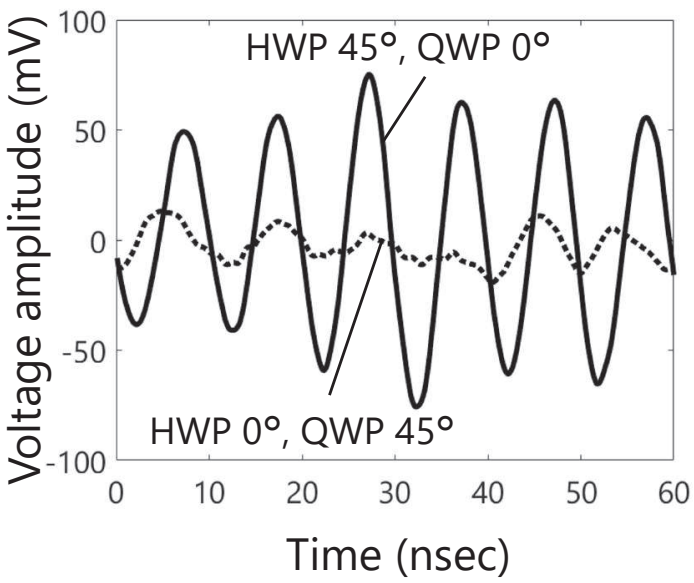

Fig. 6 Observed heterodyne beat signal. The solidline shows the case of HWP $45^{\circ}$ and QWP $0^{\circ}$, and thedashed line shows the case of HWP $0^{\circ}$ and QWP $45^{\circ}$.

when the Jones matrix represented the SOP of the transmission fiber with $(\phi, \theta)=(\pi, \pm \pi / 2)$, the carrier from 40 - Gbit/s DP-QPSK-PCTWs disappeared because of the cancellation between the extracted carriers from the X-ch. and Y-ch. PCTWs. This phenomenon was also replicated in the experiment of the carrier extraction from 10- Gbit/s DP-BPSKPCTWs. These results showed that the numerical results were confirmed by the experimental results, and that polarization dependence existed on the carrier extraction from DP-PCTWs in the SS-OPLL. The polarization dependence is the issue for the pump phase-locking to DP-PCTWs. However, there is some possibility of carrier extraction from DP-PCTWs with an arbitrary polarization by using a polarization modulator, which is controlled in accordance with extracted carrier power, in front of a carrier extractor.

\section{Acknowledgment}

Part of this research uses results of the "R\&D on Optical Signal Transmission and Amplification with Frequency/Phase Precisely Controlled Carrier" commissioned by the National Institute of Information and Communications Technology (NICT) of Japan. The authors would like to also thank NTT Device Technology Laboratories for supply of PPLN waveguides used in the experiment. 


\section{References}

1) M. Vasilyev: Opt. Express 13 (2005) 7563.

2) R. Tang, J. Lasri, P. S. Devgan, V. Grigoryan, P. Kumar, and M. Vasilyev: Opt. Express 13 (2005) 10483.

3) Y. Tian, Y.-K. Huang, S. Zhang, P. R. Prucnal, and T. Wang: Opt. Express 21 (2013) 5099.

4) S. L. I. Olsson, B. Corcoran, C. Lundström, E. Tipsuwannakul, S. Sygletos, A. D. Ellis, Z. Tong, M. Karlsson, and P. A. Andrekson: Proc. OFC, 2012 (Los Angeles, California, 2012) OW3C. 3.

5) Y. Okamura, M. Koga, and A. Takada: IEICE Commun. Express 5 (2016) 152.
6) Y. Okamura, M. Abe, K. Kondo, Y. Ohmichi, T. Kazama, T. Umeki, M. Koga, and A. Takada: Opt. Express 24 (2016) 26300

7) Y. Okamura, M. Abe, K. Kondo, T. Okabe, T. Kazama, A. Sano, T. Umeki, R. Kasahara, and A. Takada: IEICE Commun. Express 6 (2017) 566.

8) T. Umeki, T. Kazama, O. Tadanaga, K. Enbutsu, and M. Asobe: J. Lightwave Technol. 33 (2015) 1326.

9) M. Abe, T. Kazama, T. Umeki, K. Enbutsu, Y. Miyamoto, and H. Takenouchi: Proc. ECOC, 2016 (Düsseldorf, Germany, 2016) W. 4. P1. SC2. 11.

10) K. Kikuchi: Opt. Express 22 (2014) 1971 